\title{
Infectious Rupture of a Dissecting Thoracoabdominal Aortic Aneurysm Due to Citrobacter freundii
}

\author{
Masafumi Hashimoto ${ }^{1}$, Kenji Mogi ${ }^{2}$, Manabu Sakurai ${ }^{1}$, Tomoki Sakata ${ }^{1}$, Kengo Tani ${ }^{2}$, and \\ Yoshiharu Takahara ${ }^{1}$ \\ ${ }^{1}$ Funabashi Municipal Medical Center \\ ${ }^{2}$ Funabashi Municipal Medical Center Heart and Vascular Institute
}

April 15, 2021

\begin{abstract}
Here we describe a case involving an elderly man with Citrobacter freundii-associated infectious rupture of a dissecting thoracoabdominal aortic aneurysm. We performed emergency thoracoabdominal aortic replacement using a rifampicin-soaked prosthetic graft and omental flap wrapping. The patient was discharged on postoperative day 255, although he experienced pseudomembranous enteritis and paraplegia.
\end{abstract}

Infectious Rupture of a Dissecting Thoracoabdominal Aortic Aneurysm Due to Citrobacter freundii

Masafumi Hashimoto, MD, Kenji Mogi, MD, PhD, Manabu Sakurai, MD, PhD,

Tomoki Sakata, MD, Kengo Tani, MD, and Yoshiharu Takahara, MD, PhD

Division of Cardiovascular Surgery, Funabashi Municipal Medical Center Heart and Vascular Institute, Chiba, Japan

Corresponding author: Masafumi Hashimoto, MD

ORCID iD: 0000-0003-1017-6627

Division of Cardiovascular Surgery, Funabashi Municipal Medical Center Heart and Vascular Institute, 1-21-1 Kanasugi, Funabashi, Chiba 273-8588, Japan

Tel: +81-47-438-3321, Fax: +81-47-438-7323

Email:m.hashimoto0610@gmail.com

\begin{abstract}
Here we describe a case involving an elderly man with Citrobacterfreundii -associated infectious rupture of a dissecting thoracoabdominal aortic aneurysm. We performed emergency thoracoabdominal aortic replacement using a rifampicin-soaked prosthetic graft and omental flap wrapping. The patient was discharged on postoperative day 255, although he experienced pseudomembranous enteritis and paraplegia.
\end{abstract}

\section{Key clinical message}

Citrobactor freundii, an opportunistic bacterium, can infect residual dissections in the descending aorta, and the resulting infectious aortic aneurysm could rupture in a week. 
We found that a rifampicin-soaked prosthesis and omental flap wrapping were effective methods to prevent vascular prosthesis infection.

Keywords: Ruptured aortic aneurysm, Citrobacter freundii, anatomical revascularization, omental flap wrapping

\section{Introduction}

Infectious aortic aneurysms are relatively rare and account for $0.7 \%-3.0 \%$ of all aortic aneurysms. ${ }^{1}$ However, surgical management protocols for such cases are not well defined, and postoperative infection control remains a concern.

Infectious aneurysms caused by Citrobacter freundii ${ }^{2}$ are rare. Herein, we report a case of infectious rupture of a dissecting thoracoabdominal aortic aneurysm (TAAA) caused by $C$. freundii, which was successfully treated with anatomical revascularization using a rifampicin-soaked prosthetic aortic graft and omental flap wrapping.

\section{Case Presentation}

This case report has been approved by the ethics committee of our institute. The patient provided informed consent for the publication of this report.

A 63-year-old man with complaints of fever for one week, sudden lower back pain, and hypotension was admitted to our hospital. On admission, he had a temperature of 40 , an elevated white blood cell count $\left(249 \times 10^{2} / \mu \mathrm{L}\right)$ and a C-reactive protein level of $15.6 \mathrm{mg} / \mathrm{dL}$. The patient had a history of hypertension, smoking, and had undergone replacement of the descending aorta for a dissecting aortic aneurysm six years ago. Computed tomography (CT) of the remaining thoracoabdominal aortic lesion showed no aortic dilatation (Figure 1). The CT scan also revealed an acute rupture of the adventitia and a hematoma in the retroperitoneal space (Figure 2). He was diagnosed with infectious rupture of a dissecting TAAA. Emergency thoracoabdominal aortic replacement with omental flap wrapping was performed.

Dissection of the aorta just superior to the renal artery resulted in an abscess outflow and it was into the abdominal cavity. Following complete excision of the aneurysm, the abdominal aorta and the renal artery were reconstructed using a rifampicin-soaked Gelweave Coselli Thoracoabdominal Graft (Terumo Corporation, Tokyo, Japan). The proximal side of the dissected aorta was anastomosed to the prosthetic aortic graft from the previously replaced descending aorta. Since the aortic dissection extended to both the iliac arteries, the abdominal aorta was also replaced using a Gelsoft Bifurcated Graft (Terumo Corporation, Tokyo, Japan). After weaning the patient from cardiopulmonary bypass, the omentum was passed into the retroperitoneal cavity through an incision in the ligament of Treitz and wrapped around the prosthetic aortic grafts.

C. freundii was detected in the intraoperative retroperitoneal abscess, preoperative and postoperative blood cultures. The culture produced cephalosporinase (AmpC) and showed innate resistance to various penicillin antibiotics, such as ampicillin and early-generation cephalosporins.

There was no preoperative nerve paralysis; however, the patient developed paraplegia on postoperative day one. Spinal drainage showed no improvement in the patient's symptoms. Long-term carbapenem antibiotics were required to treat the AmpC-producing $C$. freundii; however, the patient consequently developed pseudomembranous enteritis caused by Clostridioides difficile. Vancomycin and Fidaxomicin were used to treat C. difficile. The patient was discharged on postoperative day 255. The blood culture at discharge was negative. A CT scan performed at the time of discharge showed no anastomotic complications or retroperitoneal fluid retention (Figure 3). However, the paraplegia persisted.

\section{Discussion}

The morbidity and mortality rates of infectious aortic aneurysm rupture are extremely high, with a poor prognosis for non-operative cases. ${ }^{3}$ The causes of infectious aneurysms are as follows: bacterial aneurysms 
caused by an infectious embolus due to endocarditis; arteritis followed by an aneurysm due to bacterial invasion of an arteriosclerotic blood vessel wall; infection of a preexisting aneurysm; and traumatic, infectious pseudoaneurysms including those caused iatrogenically. ${ }^{4}$ Our patient had arteriosclerosis and a chronic dissecting lesion, which served as a nidus of infection. In absence of a preexisting aneurysm, he was diagnosed with arteritis-type aneurysm formation.

Salmonella is the most common causative organism for this type of infection, followed by Staphylococci andStreptococci.$^{5}$ In this case, AmpC-producing C. freundii was the causative organism, and required longterm administration of carbapenem antibiotics. However, the antibiotics caused pseudomembranous enteritis due to $C$. difficile, which prolonged the duration of treatment. We administered Vancomycin and Fidaxomicin to treat the $C$. difficile ; Fidaxomicin was particularly effective in the patient. $C$. freundii is most often found in patients who are immunosuppressed; however, our patient's immune status was normal.

Prevention of infections in prosthetic aortic grafts is important in the overall treatment strategy and the following methods should be considered: 1) meticulous removal of the abscess; 2) muscle and omental flap wrapping; ${ }^{6} 3$ ) addition of antibacterial agents to the prosthetic grafts, (e.g., rifampicin soaking); ${ }^{7} 4$ ) the use of a homograft; ${ }^{8}$ and 5) extra-anatomical revascularization. ${ }^{1}$ In such an emergency situation it was impractical to obtain a homograft. Extra-anatomical reconstruction was also non-viable for a TAAA requiring reconstruction of the major abdominal branch. Therefore, methods 1-3 were used to avoid infection of the grafts. If complete removal of the arterial wall containing the infected tissue is intended in infectious TAAA, reconstruction of the intercostal artery is not viable, and spinal cord ischemia is a concern. In this case, widespread infection made it difficult to reconstruct the intercostal artery and consequently led to paraplegia.

\section{Conclusion}

We encountered a rare case of infectious TAAA due to $C$. freundiiinvasion of a preexisting dissecting aortic lesion, which led to aortic rupture within a week. AmpC production by the organism required administration of carbapenem antibiotics that resulted in pseudomembranous enteritis. The findings suggest that $C$. freundii , an opportunistic bacterium, can infect residual dissections in the descending aorta, and the resulting infectious aortic aneurysm could rupture in a week. A rifampicin-soaked prosthesis and omental flap wrapping may be effective methods to prevent vascular prosthesis infection.

Acknowledgments: The authors received no financial support for the research, authorship and publication of this article.

Conflict of Interest Statement: The authors have no conflict of interest to declare.

\section{Author contributions :}

Masafumi HASHIMOTO: Corresponding author.

Kenji MOGI: Approval of the manuscript.

Manabu SAKURAI: Approval of the manuscript.

Tomoki SAKATA: Drafting of the manuscript.

Kengo TANI: Data collection.

Yoshiharu TAKAHARA: Critical revision of the manuscript.

\section{References}

1. Müller, B. T., Wegener, O. R., Grabitz, K., Pillny, M., Thomas, L., and Sandmann, W. 2001. Mycotic aneurysms of the thoracic and abdominal aorta and iliac arteries: experience with anatomic and extraanatomic repair in 33 cases. J Vasc Surg 33:106-113. 
2. Kwon, T. W., Kim, H. K., Moon, K. M., Cho, Y. P., and Park, S. J. 2010. In situ polytetrafluoroethylene graft bypass for primary infected aneurysm of the infrarenal abdominal aorta. World J Surg 34:1689-1695.

3. Hsu, R. B., Chen, R. J., Wang, S. S., and Chu, S.H. 2004. Infected aortic aneurysms: clinical outcome and risk factor analysis. J Vasc Surg 40:30-35.

4. Redy, D. J., and Ernst, C. B. Infected aneurysms. In: Rutherford RB (Ed). Vascular Surgery. Philadelphia, PA: W. B. Saunders Company; 2000. 1383-1397 p.

5. Fichelle, J. M., Tabet, G., Cormier, P., Farkas, J. C., Laurian, C., Gigou, F., Marzelle, J., Acar, J., and Cormier, J. M. 1993. Infected infrarenal aortic aneurysms: when is in situ reconstruction safe? J Vasc Surg 17:635-645.

6. Mathisen, D. J., Grillo, H. C., Vlahakes, G. J., and Daggett, W. M. 1988. The omentum in the management of complicated cardiothoracic problems. J Thorac Cardiovasc Surg 95:677-684.

7. Hayes, P. D., Nasim, A., London, N. J., Sayers, R. D., Barrie, W. W., Bell, P. R., and Naylor, A. R. 1999. In situ replacement of infected aortic grafts with rifampicin-bonded prostheses: The Leicester experience (1992 To 1998). J Vasc Surg 30:92-98.

8. Berdat, P. A., Malinverni, R., Kipfer, B., and Carrel, T. P. 1998. Homograft failure in mycotic aortic aneurysm caused byStreptococcus pneumoniae. Ann Thorac Surg 66:1818-1819.

\section{Figure Legends}

Figure 1. A computed tomography angiogram does not show aortic dilatation. The dissection remaining in the thoracoabdominal aortic lesions has a maximum diameter of $38 \mathrm{~mm}$. (a) At the level of the superior mesenteric artery level, (b) at the level of the renal arteries, (c) infrarenal level, and (d) at the level of the terminal aorta.

Figure 2. Computed tomography angiogram reveals a rupture of the adventitia and a hematoma in the retroperitoneal cavity. (a) At the level of the superior mesenteric artery, (b) at the level of the renal arteries, (c) infrarenal level, and (d) at the level of the terminal aorta. This computed tomography angiogram was obtained one week after the computed tomography angiogram (Figure 1) was taken.

Figure 3. Postoperative computed tomography scan obtained at the time of discharge. (a, b) The scan shows the retroperitoneal space without fluid retention and (c) without any anastomotic complications.

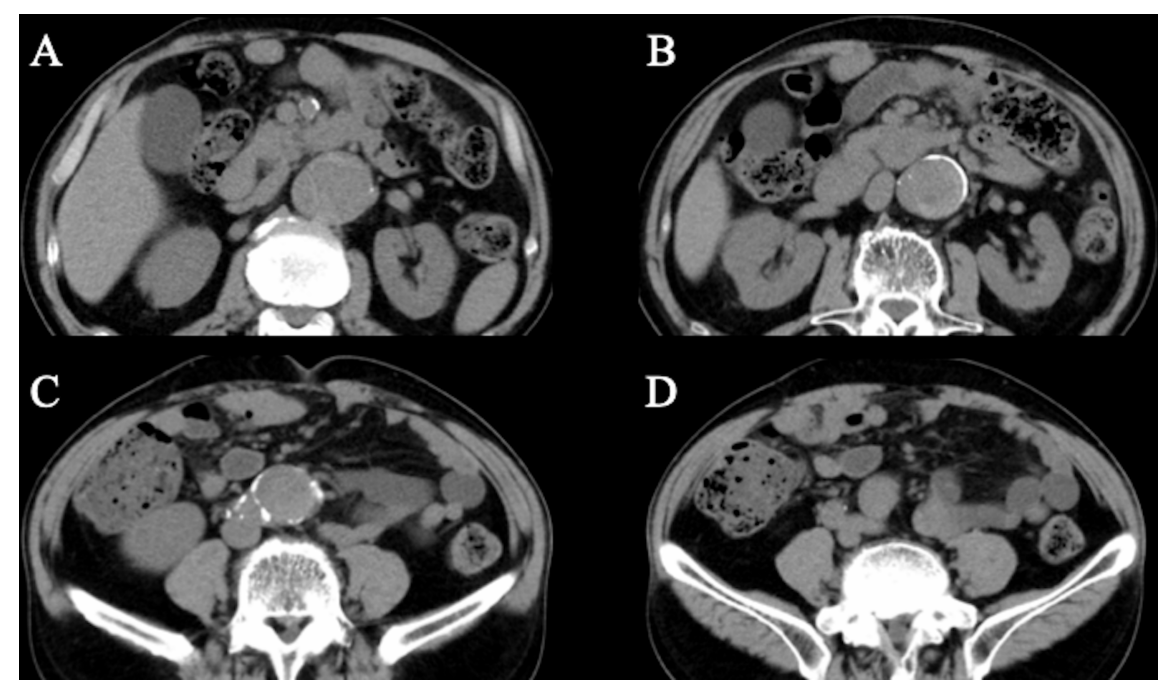



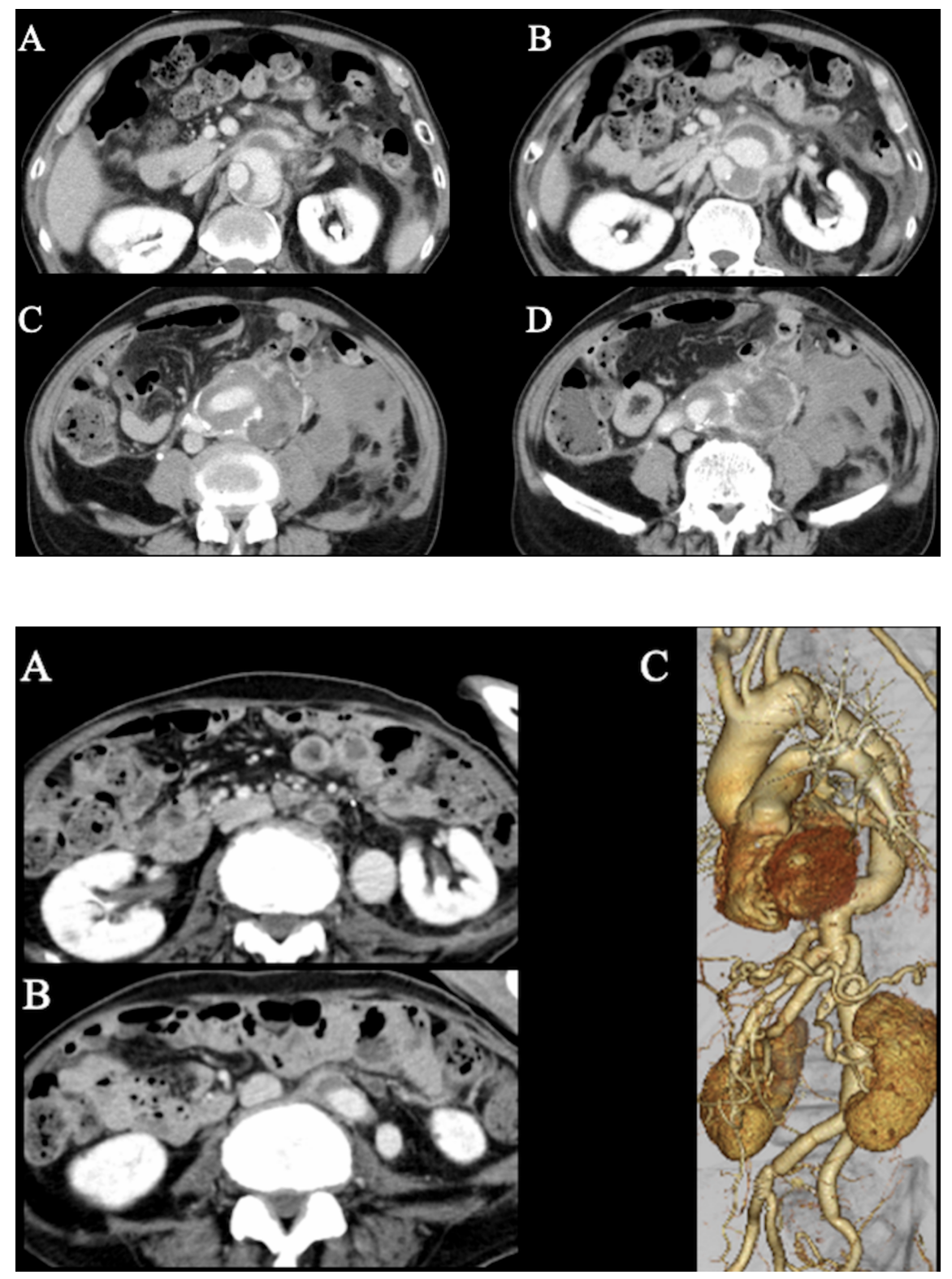